\title{
Acute panmyelosis with myelofibrosis: an entity distinct from acute megakaryoblastic leukemia
}

\author{
Attilio Orazi ${ }^{1}$, Dennis P O’Malley ${ }^{1}$, Jiazhong Jiang ${ }^{1}$, Gail H Vance ${ }^{2}$, Janice Thomas $^{1}$, \\ Magdalena Czader ${ }^{1}$, Wei Fang ${ }^{1}$, Caroline $\mathrm{An}^{1}$ and Peter M Banks ${ }^{3}$ \\ ${ }^{1}$ Department of Pathology and Laboratory Medicine, Indiana University School of Medicine, Indianapolis, \\ Indiana, USA; ${ }^{2}$ Department of Medical and Molecular Genetics, Indiana University School of Medicine, \\ Indianapolis, Indiana, USA and ${ }^{3}$ Carolinas Medical Center, Charlotte, NC, USA
}

\begin{abstract}
The WHO criteria for diagnosing acute panmyelosis with myelofibrosis are somewhat distinct from those for acute megakaryoblastic leukemia. However, clinical and hematopathologic findings partially overlap. This has raised questions as to whether these are indeed separate, definable entities. To determine the potential importance of bone marrow biopsy supplemented by immunohistochemistry in distinguishing between these two conditions, we studied 17 bone marrow biopsies of well-characterized cases of acute panmyelosis with myelofibrosis (six cases) and acute megakaryoblastic leukemia (11 cases). We compared blast frequency, reticulin content, CD34 expression, and the degree of megakaryocytic differentiation of the blast cells in these two conditions. Our results demonstrate important differences. Acute panmyelosis with myelofibrosis is characterized by a multilineage myeloid proliferation with a less numerous population of blasts than acute megakaryoblastic leukemia $(\boldsymbol{P}<0.01)$. In the former condition, blasts are always positive with CD34, while in acute megakaryoblastic leukemia they express CD34 in $60 \%$ of the cases. The blasts in acute panmyelosis with myelofibrosis only rarely express megakaryocytic antigens. By contrast, acute megakaryoblastic leukemia has a significantly higher proportion of blasts expressing megakaryocytic antigens $(P<0.01$ with $C D 42 b)$. Our results confirm that histology supplemented by immunohistochemistry permits the distinction of these conditions in routinely processed bone marrow biopsies.
\end{abstract}

Modern Pathology (2005) 18, 603-614, advance online publication, 3 December 2004; doi:10.1038/modpathol.3800348

Keywords: acute panmyelosis myelofibrosis; acute megakaryoblastic leukemia

In 1963, Lewis and Szur ${ }^{1}$ described a disorder that they termed 'malignant myelosclerosis,' characterized by an acute clinical course, rapid development of peripheral blood cytopenias with leukoerythroblastosis, and no detectable splenomegaly. Bone marrow findings in their study were considered to be identical to those in classic chronic idiopathic myelofibrosis (CIMF). A number of subsequent studies have described a similar disorder that has been termed 'acute myelofibrosis,' 'acute myelosclerosis,' 'acute megakaryocytic myelofibrosis,' and 'acute myelodysplasia with myelofibrosis. ${ }^{2-5}$ Most of these cases are now believed to correspond to the

Correspondence: Dr A Orazi, MD, FRCPath (Engl.), Department of Pathology and Laboratory Medicine, Indiana University School of Medicine, 702 Barnhill Drive RI 0969, Indianapolis, IN 46202-5200, USA

E-mail: aorazi@iupui.edu

Received 30 June 2004; revised and accepted 11 October 2004; published online 3 December 2004 entity presently defined as acute panmyelosis with myelofibrosis (APMF) in the WHO classification system of hematopoietic malignancies. ${ }^{6}$ APMF is exceedingly rare and corresponds to less than $1 \%$ of the cases of acute myeloid leukemias (AML), although this may be an underestimation due to missed diagnosis. The WHO criteria for the diagnosis of APMF include: panmyelosis, significant marrow fibrosis, pancytopenia, normal erythrocyte morphology, lack of splenomegaly, and a rapidly fatal course. ${ }^{7}$

Most cases of APMF are characterized by a fibrotic bone marrow with an increased number of immature trilineage hematopoietic elements, and conspicuously dysplastic megakaryocytes predominately of small size showing variable degrees of atypia including the presence of hypolobulated or nonlobulated nuclei. Foci of blasts are found scattered throughout the marrow. The overall frequency of blasts in APMF marrows is uncertain; however, its precise determination is not considered a diagnostic 
requirement, according to the WHO system. The peripheral blood in APMF usually shows pancytopenia with absent or only rare circulating blasts and lacks the anisocytosis and poikilocytosis of CIMF. Splenomegaly is minimal or absent, and myeloid metaplasia in the spleen is not a prominent feature. The course is rapidly fatal, often terminating with an overtly leukemic myeloid phase, or rarely with a lymphoblastic malignancy. In the terminal stage, splenomegaly may be observed, which usually results from leukemic infiltration of the red pulp. ${ }^{8-10}$ The disease usually occurs in adults, but rare cases have been described in children. ${ }^{7}$

Acute megakaryoblastic leukemia (AMKL) is a rare form of AML and accounts for $3-5 \%$ of all myeloid leukemias. ${ }^{11}$ AMKL is diagnosed according to criteria of the WHO system, which closely mirrors those of the earlier FAB classification previously established for this subtype of AML. ${ }^{12}$ The main requirement is a minimum of $20 \%$ blasts, the majority of which showing megakaryocytic differentiation, usually demonstrated by flow cytometry. ${ }^{7}$ Additional morphologic features seen in a variable proportion of cases of AMKL include the presence of abnormal small megakaryocytes with hypolobulated or nonlobulated nuclei and a marked degree of reticulin fibrosis, findings that overlap both APMF and AMKL.

As the distinction between APMF and AMKL is difficult, we tested the ability of various morphologic and immunohistochemical criteria to distinguish the two entities. In addition, we analyzed cytogenetic, flow cytometric, and clinical results in relation to the two entities.

\section{Materials and methods}

We evaluated formalin-fixed, nitric acid decalcified, paraffin-embedded bone marrow biopsies obtained from the pathology files of the Department of Pathology, Indiana University School of Medicine/ Clarian Health Partners, Indianapolis, Indiana. All these cases were consecutively diagnosed within a period of approximately 10 years. Additionally, five cases of AMKL were obtained from the consultation files of one author (PMB).

The diagnostic categorization of these cases was based on a comprehensive multiparametric evaluation, which included a combination of clinical characteristics, morphology, immunohistochemistry, and when available, flow cytometry and cytogenetics, in accordance with the diagnostic approach outlined in the current WHO classification. ${ }^{6,11}$ In each case, H\&E-, Giemsa-, and reticulin-stained sections were reviewed. A Wright-stained peripheral blood smear was evaluated in each case. When available, bone marrow aspirate smears and touch preparations (of bone marrow biopsy) were also examined. All cases included in this study were 'de novo' myeloid neoplasms; none of the AMKL cases was associated with Down's syndrome or history of mediastinal germ cell tumor.

\section{Histologic Evaluation}

Core biopsies were reviewed for the following characteristics: cellularity, blast count, and the presence and quality of trilineage hematopoietic elements. The percentage of blasts was determined in each case by a careful analysis of H\&E- and/or Giemsa-stained bone marrow biopsy sections which were cut at $3 \mu \mathrm{m}$. A 500-cell differential count was performed in each case. In APMF blasts were defined as cells with high nuclear:cytoplasmic ratio, dispersed nuclear chromatin, and scanty cytoplasm. A comparison 'side by side' with a corresponding section stained with CD34 (see below) was particularly helpful in confirming the marrow blast percentage. In AMKL, blasts were identified on the basis of their cytologic characteristics, which included, in poorly differentiated cases, a lymphoblast-like appearance with coarse to variably dispersed chromatin and scanty cytoplasms. In more differentiated cases, megakaryoblasts with a lower nuclear:cytoplasmic ratio and more abundant cytoplasm were observed. Similarly to APMF, a comparison with sections immunostained with CD42b and CD34 (see below) was also performed in these cases. This was helpful in confirming the estimated marrow blast cell percentage.

The lineage of the blasts was determined by immunohistochemical staining (see below). Particular emphasis was given to the morphologic evaluation of megakaryocytes. Megakaryoblasts, micromegakaryocytes (cell diameter $<15 \mu \mathrm{m}$ ), and 'atypical' megakaryocytes (hypolobulated forms larger than micromegakaryocytes) were distinguished. The morphologic definition of these forms is based on the previously published terminology and criteria. ${ }^{13-15}$

In addition, the degree of reticulin fibrosis was evaluated in each case. The grading of bone marrow reticulin fibrosis was performed according to the system originally proposed by Manoharan et $a l^{16}$ on a scale of $0-3+$. It should be noted that $4+$ fibrosis (presence of mature collagen) can be established only by the application of a trichrome stain; this was not available in this study.

\section{Flow Cytometry-Methods}

Bone marrow aspirates or minced bone marrow core biopsy material was analyzed using three-color flow cytometry. After red blood cell depletion with ammonium chloride and subsequent washes, a total cell count was determined using an automated cell counter. The viability of the sample was analyzed with propidium iodide staining. Following these initial steps, cell suspensions were incubated with antibody cocktails. Antibodies used were specific to CD14, CD5, CD19, CD10, CD2, CD7, CD3, HLA-DR, 
CD61, glycophorin, CD33, CD34, CD16, CD56, CD64, CD41, CD22, CD117, CD13, CD15, myeloperoxidase, TdT, and kappa and lambda light chains. The antibodies were directly conjugated with fluorescein isothiocyanate (FITC), phycoerythrin (PE), or rhodamine (RD). Flow cytometric analysis was performed using a COULTER ${ }^{\circledR}$ EPICS $\mathrm{XL}^{\mathrm{TM}}$ flow cytometer (Beckman Coulter, Miami, FL, USA). Cells of interest were gated on right angle scatter vs CD45 display. An antigen was considered positive if $20 \%$ or more cells in the blast gate stained with a specific antibody.

\section{Immunoperoxidase Methods}

The following immunoperoxidase stains were performed using an automated immunostainer (DAKO, Carpinteria, CA, USA) and a standard streptavidinbiotin-peroxidase complex technique. The progenitor/early precursor associated antibody (Ab) CD34 (QBEND10) and the following anti-Mk Abs: CD31 (JC70A), CD42b (MM2/174), vWF (polyclonal factor VIII), and anti-LAT (linker for activation of T cells; antibody kindly provided by Professor George Delsol, see Acknowledgments). Myeloid and erythroid differentiation was assessed by using polyclonal antimyeloperoxidase and antihemoglobin antibodies (both obtained from DAKO). Briefly, endogenous peroxidase activity was blocked with $3 \%$ hydrogen peroxide in methanol, and endogenous biotin activity was blocked using avidin and biotin. The peroxidase activity was developed with 3,3-diaminobenzidene (DAB), and counterstained with hematoxylin. Controls stained appropriately. The immunohistochemical results were expressed as mean $( \pm$ s.d.) percentage of marrow blasts reactive with the given antibody.

\section{Cytogenetic Methods}

For bone marrow aspirate specimens, the cell culture, harvesting and GTG-banded karyotype methods were performed as previously described. ${ }^{17}$ In one case, a bone core biopsy specimen was first minced with dissecting scissors and then cultured similar to bone marrow aspirate specimens (patient 13).

\section{Results}

The patients in this study ranged in age from 2 to 84 years; 10 were males and seven females. The median age was 72 (mean $70.6 \pm 8.5$ ) years in APMF and 57 years (mean $55.0 \pm 22.3$ ) in AMKL patients. The most common symptoms at presentation included fatigue, fever, bruising, and shortness of breath. Hepatosplenomegaly was noted in two patients with AMKL and in none of the cases classified as APMF. Survival data were available for all six APMF cases and for seven (of 11) AMKL patients.
Table 1 Clinical features of cases

\begin{tabular}{lcccl}
\hline & Case number & Age & Gender & Survival \\
\hline APMF & 1 & 74 & M & D-27 days \\
& 2 & 79 & F & D-1 month, 5 days \\
& 3 & 57 & M & D-16 months \\
& 4 & 70 & M & D-14 days \\
& 5 & 65 & M & AWD-31 months \\
& 6 & 79 & M & D-32 days \\
AMKL & 7 & 45 & M & NA \\
& 8 & 36 & F & NA \\
& 9 & 54 & M & D-19 days \\
& 10 & 2 & F & D-11 months, 21 days \\
& 11 & 70 & F & NA \\
& 12 & 57 & F & D-5 days \\
& 13 & 76 & M & NA \\
& 14 & 62 & F & D-3 months \\
& 15 & 84 & M & D-2 months, 5 days \\
& 16 & 52 & F & AWD-27 months \\
& 17 & 67 & M & D-30 days \\
\hline
\end{tabular}

$\mathrm{D}=$ dead $\mathrm{AWD}=$ alive with disease $\mathrm{NA}=$ result not available

In APMF, survival from diagnosis to death (5/6 patients) ranged from 14 to 480 days, with a median value of 32 days. The single patient who died at 480 days was treated with chemotherapy followed by allogeneic bone marrow transplant; the cause of death was a disseminated fungal infection 4 weeks after the transplant. At autopsy no evidence of leukemia was found. None of the other patients in the APMF group received bone marrow transplant. One patient, treated with chemotherapy, was alive with disease at 930 days follow-up.

In the AMKL group, the survival from diagnosis to death (six patients) was comprised between 5 and 351 days with a median value of 47.5 days. One patient was alive with disease at 540 days from the diagnosis. Three patients in the AMKL group received bone marrow transplants. No significant differences were observed between the two diagnostic groups. The clinical features are summarized in Table 1.

\section{Peripheral Blood Results}

All patients with APMF and most patients with AMKL showed pancytopenia. The degree of leukopenia and the lowest absolute neutrophil counts were seen in cases of APMF (see Table 2). Unremarkable red cell morphology was observed in six cases of APMF; one case showed mild anisocytosis. The neutrophils showed dysgranulopoietic changes including the presence of pseudo Pelger-Huet and/ or hypogranulated forms in four of six $(67 \%)$ of APMF and in two of $11(18 \%)$ of the AMKL cases. Abnormal platelets, including hypogranular, hypergranular, and giant forms, were occasionally observed in both groups. In addition, fragments of megakaryocytic cytoplasm and platelet clumps were 
Table 2 Comparison of results of APMF vs AMKL

\begin{tabular}{|c|c|c|c|c|c|c|c|c|c|}
\hline & \multirow{2}{*}{$\begin{array}{c}\text { Case } \\
\text { no. }\end{array}$} & \multirow[t]{2}{*}{$W B C(A N C) 10^{9} / 1$} & \multirow{2}{*}{$\begin{array}{c}\text { PB blast } \\
(\%)\end{array}$} & \multirow{2}{*}{$\begin{array}{c}\text { BM blasts } \\
\quad(\%)\end{array}$} & \multirow[t]{2}{*}{ Retic } & \multicolumn{4}{|c|}{ Percentage of BM blasts positive with given antibody } \\
\hline & & & & & & $\%$ CD34+ & $\% C D 42+$ & $\% v W F$ & $\% C D 31+$ \\
\hline \multicolumn{10}{|l|}{$A P M F$} \\
\hline & 1 & $1.96(0.7)$ & 11 & 25 & 2 & 60 & 10 & 10 & 51 \\
\hline & 2 & $8.60(2.3)$ & 0 & 20 & 3 & 63 & 5 & 5 & 40 \\
\hline & 3 & $1.1(0.2)$ & 0 & 10 & 3 & 80 & 10 & 11 & 70 \\
\hline & 4 & 3.04 (1.3) & 33 & 25 & 3 & 60 & 20 & 12 & 60 \\
\hline & 5 & $2.27(0.9)$ & 1 & 20 & 2 & 70 & 10 & 5 & 15 \\
\hline & 6 & $2.0(0.6)$ & 3 & 25 & 3 & 60 & 8 & 6 & 90 \\
\hline Mean & & 3.2 (ANC: $1.0 \pm 0.7$ ) & 8 & 20.8 & 2.6 & 65.5 & 10.5 & 8.1 & 54.3 \\
\hline ( \pm s.d. $)$ & & $( \pm 2.7)$ & $( \pm 12.9)$ & $( \pm 5.8)$ & $( \pm 0.5)$ & $( \pm 8.0)$ & $( \pm 5.0)$ & $( \pm 3.1)$ & $( \pm 25.7)$ \\
\hline Median & & 2.1 (ANC: 0.8 ) & 2 & 22.5 & 3 & 61.5 & 10 & 8 & 55.5 \\
\hline \multirow[t]{11}{*}{ AMKL } & 7 & $1.3(1.2)$ & 1 & 85 & 2 & 80 & $\mathrm{NE}$ & $\mathrm{NE}$ & 90 \\
\hline & 8 & 4.8 (NA) & 10 & 50 & 3 & 80 & 80 & 5 & 30 \\
\hline & 9 & $8.8(4.0)$ & 8 & 80 & 3 & 5 & 35 & $\mathrm{NE}$ & 10 \\
\hline & 10 & $29.5(5.3)$ & 38 & 80 & 3 & 4 & 80 & 20 & 90 \\
\hline & 11 & $13.6(7.3)$ & 9 & 70 & 2 & 5 & 100 & 80 & $\mathrm{NE}$ \\
\hline & 12 & $1.9(0.1)$ & 30 & 60 & 3 & 70 & 30 & 10 & 90 \\
\hline & 13 & 1.2 (NA) & NA & 90 & 3 & 80 & 70 & $\mathrm{NE}$ & 50 \\
\hline & 14 & $9.7(2.7)$ & 20 & 70 & 3 & 70 & 40 & 20 & 50 \\
\hline & 15 & $1.1(0.4)$ & NA & 20 & 3 & 60 & 25 & 10 & 80 \\
\hline & 16 & $6.7(3.7)$ & 50 & 20 & 3 & 5 & 50 & 70 & 90 \\
\hline & 17 & $8.1(1.5)$ & 37 & 30 & 3 & 80 & 30 & 40 & 90 \\
\hline Mean & & $7.8($ ANC $2.9 \pm 2.4)$ & 28.3 & 63.1 & 2.7 & 43.5 & 55.4 & 28.8 & 62.7 \\
\hline ( \pm s.d.) & & $( \pm 8.2)$ & $( \pm 16.8)$ & $( \pm 25.9)$ & $( \pm 0.5)$ & $( \pm 35.6)$ & $( \pm 26.4)$ & $( \pm 28.7)$ & $( \pm 29.8)$ \\
\hline Median & & 6.7 (ANC: 2.7 ) & 20 & 70 & 3 & 70 & 45 & 20 & 85 \\
\hline
\end{tabular}

${ }^{\mathrm{a} B o n e}$ marrow blast count obtained on bone marrow biopsy.

$\mathrm{PB}=$ peripheral blood; $\mathrm{BM}=$ bone marrow; $\mathrm{NE}=$ nonvaluable due to poor immunoreactivity of the sample; $\mathrm{NA}=$ result not available. Statistical results (Student's $t$-test): APMF vs AMKL, percentage of bone marrow blasts, $P<0.01$; percentage of CD42-positive blasts, $P<0.01$. Other results: NS.

Note: LAT staining performed satisfactorily on only 10 cases; the results observed paralleled CD42b. However, the result was statistically NS.

rarely seen in both groups. Rare circulating blasts were present in 3/6 cases of APMF; in APMF, only $2 / 6$ patients had $>10 \%$ circulating blasts. Six (of nine) cases of AMKL showed a frankly leukemic picture with $\geqslant 10 \%$ blasts; only rare blasts were seen in 3/9 cases. The blasts both in APMF and AMKL appeared poorly differentiated. In one patient with AMKL cytoplasmic blebs were occasionally seen.

\section{Bone Marrow Results}

\section{Bone marrow aspirate smears and/or touch preparations}

Marrows were inaspirable in all cases of APMF ('dry tap'). The marrow biopsy touch preparations available in three cases showed occasional blasts and dysplastic megakaryocytes in $2 / 3$ cases (Figure 1a). In AMKL, 9/11 cases had inadequate or no aspirate for review. In the two aspirable cases, sheets of poorly differentiated blasts with occasional cytoplasmic blebs were noted (Figure 2a).

\section{Bone marrow biopsy}

Bone marrow was hypercellular in all biopsies of both APMF and AMKL. In APMF, all cases showed variable degrees of expansion of erythroid, myeloid, and megakaryocytic precursors (Figure 1b, c). In three cases, erythropoiesis was markedly increased with numerous immature erythroid precursors. An increased number of micromegakaryocytes and atypical megakaryocytes with nonlobulated or hypolobulated nuclei were always present (Figure 1c). These cells were easily recognizable in sections immunostained with CD42b (Figure 1d), LAT (Figure 1e), or vWF. vWF, however, often showed a high nonspecific background staining. CD31, while similarly positive, was occasionally difficult to interpret due to the staining of other types of (nonmegakaryocytic) myeloid cells, macrophages/ monocytes in particular. In all APMF cases, an increased number of blasts often arranged into scattered aggregates and clusters were detected. In none of the cases, the percentage of blasts was higher than $25 \%$. Case 3 had the lowest number of blasts $(10 \%)$, a value which, if considered by itself, would make the case fit best within a myelodysplastic syndrome (MDS), refractory anemia with excess of blasts (RAEB) category. However, the original diagnosis of APMF was maintained in virtue of the combination in this case of abrupt clinical onset, a striking degree of reticulin fibrosis, and the abundance and prominence of small megakaryocytes 

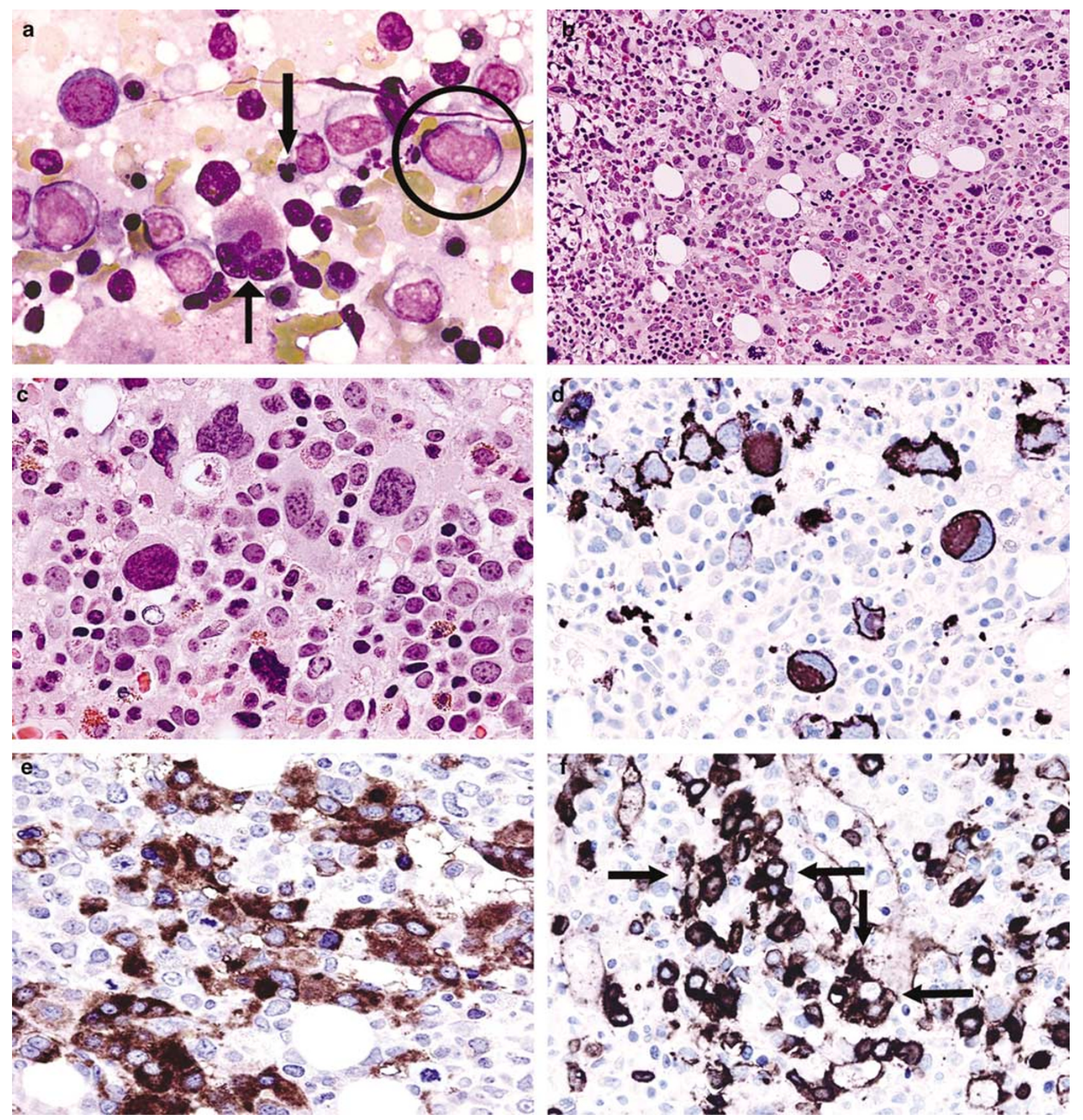

Figure 1 Acute panmyelosis with myelofibrosis. (a) Bone marrow biopsy touch preparation from a patient with APMF (patient 5). In the photo note a myeloblast (circled), a trilobated dysplastic erythroblast (arrowed), and a small dysplastic megakaryocyte. (b, c) Bone marrow biopsy sections from patient with APMF (patient 1). The marrow shows high cellularity due to a multilineage proliferation (panmyelosis). Note the presence of numerous hypolobulated dysplastic megakaryocytes with disperse chromatin pattern and an excess of blasts. Although the number of marrow blasts is always increased in cases of APMF, they never represented the majority of the marrow cellularity. (d, e) Immunoperoxidase stains for CD42b (d) and LAT (e). Both markers are positively expressed in the megakaryocytes and highlight the predominance of small size abnormal forms that characterized cases of APMF. (f) Immunoperoxidase reaction with antibody to CD34. The slide shows numerous CD34-positive blasts that are arranged into clusters and aggregates (arrows). The CD34 stains the majority of the blasts in cases of APMF. (g, h) Sections of bone marrow biopsies of patients with APMF stained with antimyeloperoxidase (g) and antihemoglobin (h) antibodies. Note the multilineage nature of the precursor cells seen in APMF, which include myeloid as well as erythroid precursors. (i) Section of bone marrow biopsy of a patient with APMF stained for reticulin (Gomori stain). Note the diffuse fibrosis and its coarse fiber pattern (grade 3).

with dysplastic features including a high frequency of nonlobulated or hypolobulated nuclei with dispersed nuclear chromatin.
In all cases, the majority of the blasts expressed CD34 (Figure 1f). They were arranged in clusters easily identifiable by their CD34 reactivity. A 
608
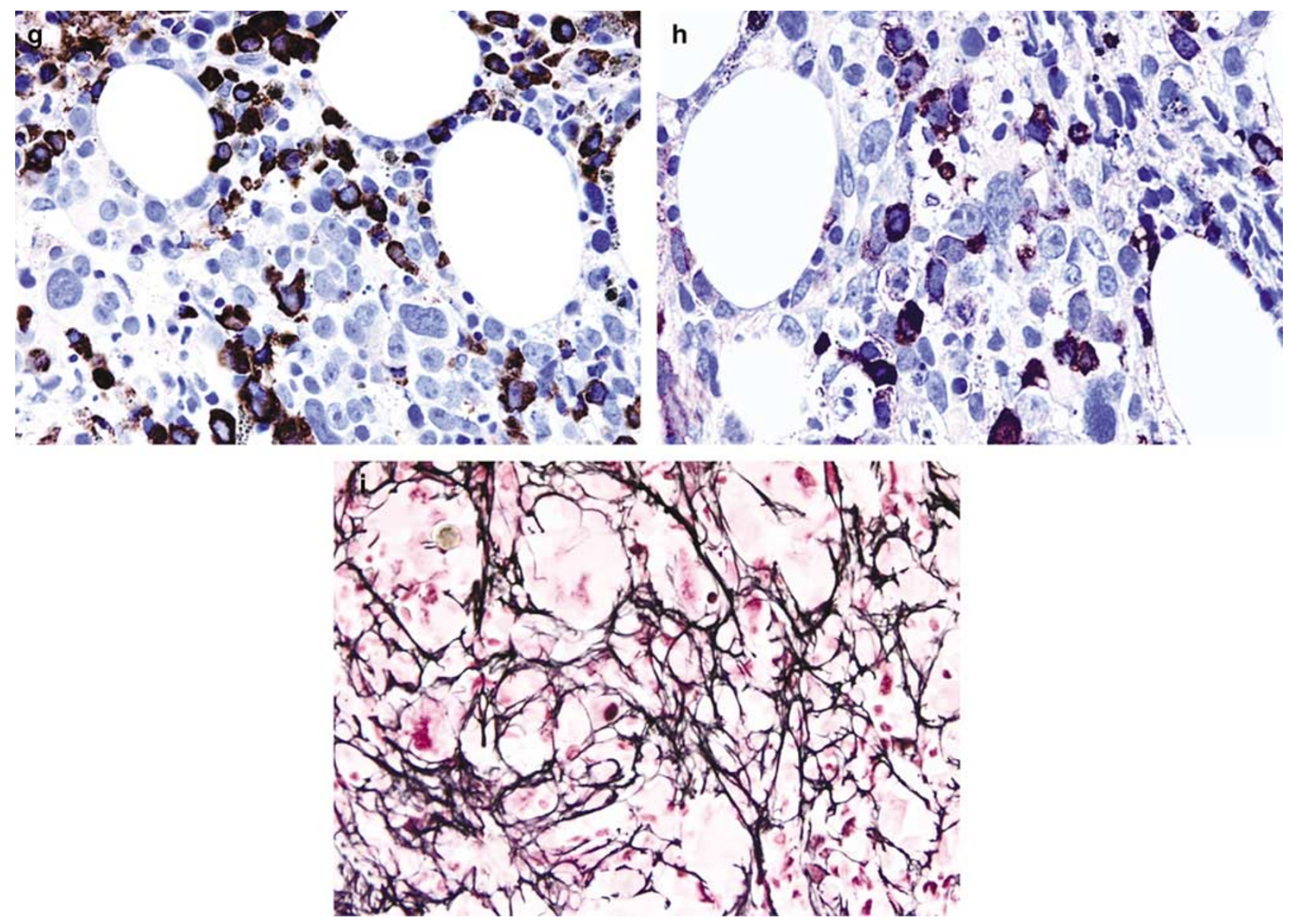

Figure 1 Continued.

variable proportion of the blasts expressed myeloperoxidase (Figure 1g). Hemoglobin (Figure 1h) reactivity facilitated the identification of erythroblasts and helped in distinguishing proerythroblasts from nonerythroid blasts in selected cases. In 5/6 APMF cases, only very rare megakaryoblasts, identified on the basis of their CD42b and/or LAT reactivity, were identified. All cases showed significant $(\geq 2+)$ degree of reticulin fibrosis. Results summarized in Table 2.

In contrast to APMF, nine of 11 cases of AMKL showed high blast counts (AMKL vs APMF, $P=<0.01$ ) (Table 2 and Figure 2a, b). A predominance of megakaryoblasts, identified by their flow cytometric characteristics and/or by their immunohistochemical reactivity with CD42b (Figure 2c),
CD31, vWF, or LAT (Figure 2d) was seen in nine of 11 cases. Three AMKL cases $(12,15,17)$ showed a low frequency of CD42b and vWF immunoreactivity but a strong CD31 staining. Case 9 expressed CD42b only and at a low level. However, in these four cases, the majority of marrow blasts expressed CD41 and/ or CD61 by flow cytometry (Table 3). Antimyeloperoxidase was consistently negative (Figure 2f) while antihemoglobin stained occasionally residual erythroblast (Figure 2g).

The most consistently positive staining of the blasts in AMKL was obtained with CD42b (Figure 2c) and LAT (Figure 2d). The difference observed with CD42b between AMKL and APMF was statistically significant $(P<0.01)$. We did not have satisfactory immunostaining in some cases by using LAT. The limited

Figure 2 (a) Bone marrow aspirate smear from a patient with AMKL. The aspirate in this case (patient 9) shows numerous poorly differentiated megakaryoblasts with only occasional pseudopods. One blast showing erythrophagocytosis is noted. (b) Bone marrow biopsy The bone marrow cavities contain large confluent aggregates of megakaryoblasts. The megakaryoblasts in this case are predominantly poorly differentiated; note the prominent nucleoli and variable amount of cytoplasm displayed by the blasts. (c, d) Immunoperoxidase stain for CD42b (c) and LAT (d). The positive reaction seen with both antibodies confirms the megakaryocytic nature of the blasts (patient 13). (e) Immunoperoxidase stain for CD34. CD34 is positive in the blasts in this case of AMKL. Note the presence of large confluent aggregates and sheets of positive blasts, similar to what is seen in other cases of acute leukemia expressing CD34 (but not in APMF). (f, g) Immunoperoxidase stain for myeloperoxidase (f) and hemoglobin (g) (patient 13). Myeloperoxidase is totally negative in the blasts. A small number of maturing erythroblasts within the scanty residual maturing bone marrow population is positively stained with antihemoglobin. 


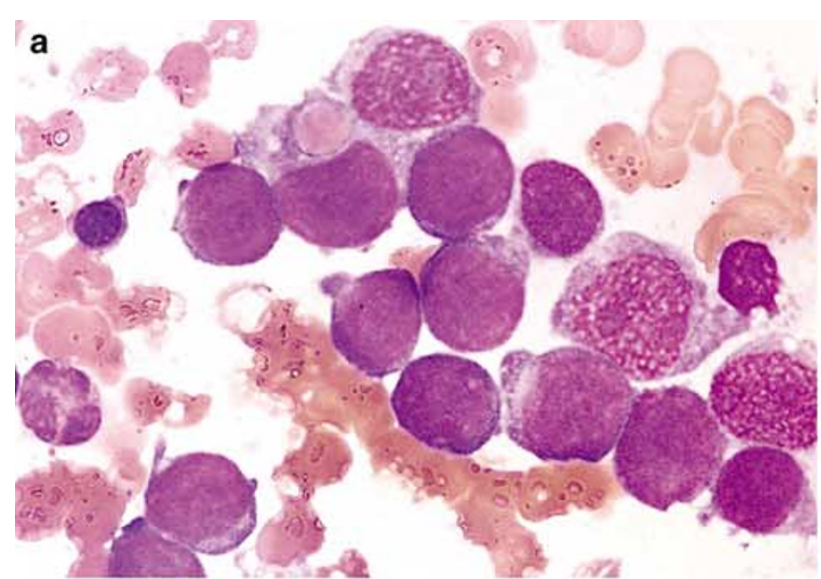

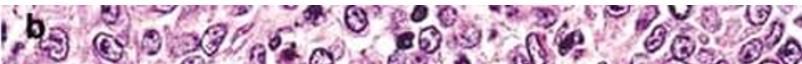

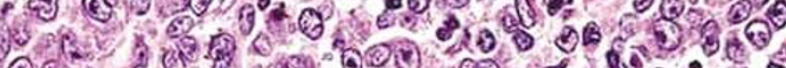

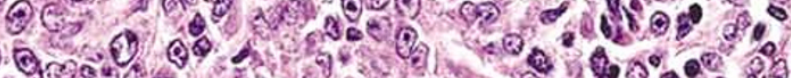

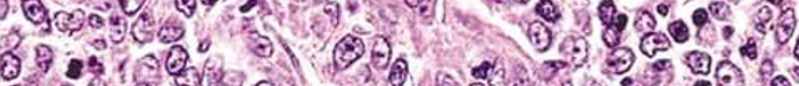
-

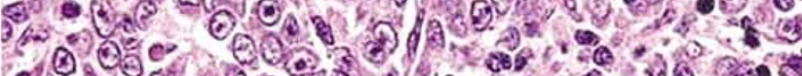

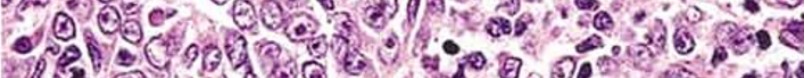
28 n.

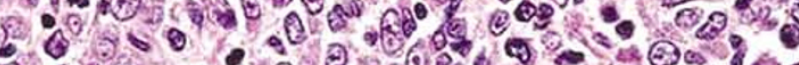

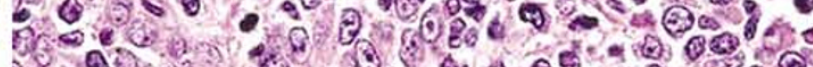

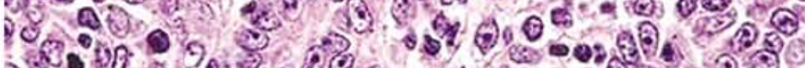

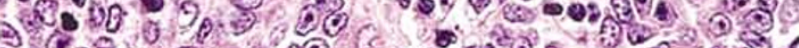

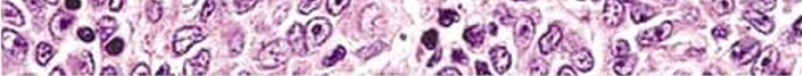

\section{(c)}

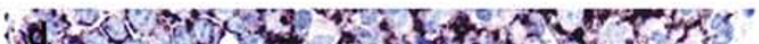

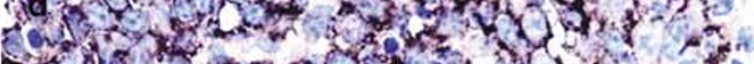

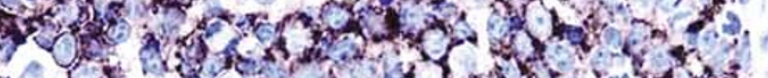

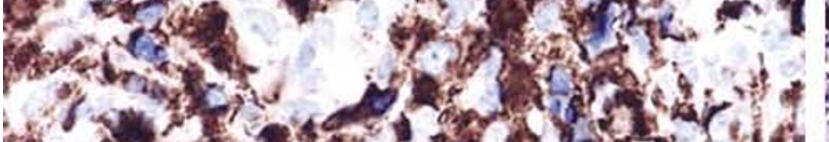

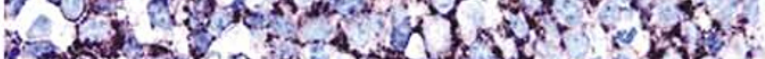

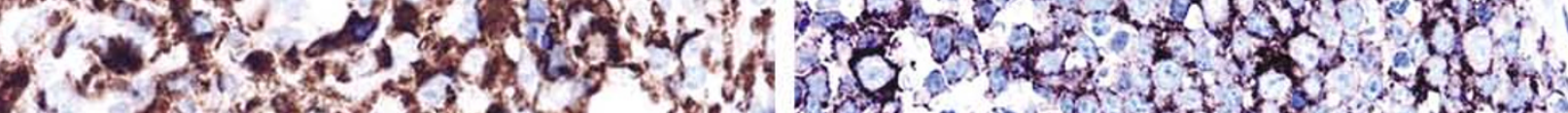

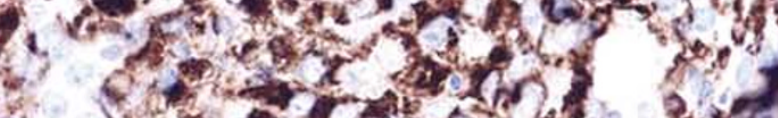

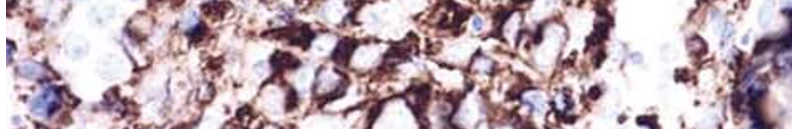

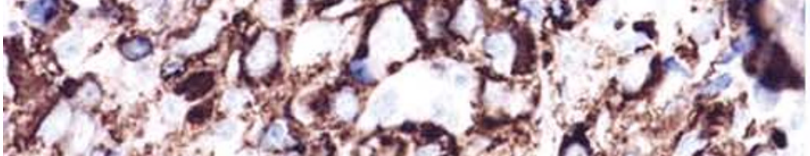

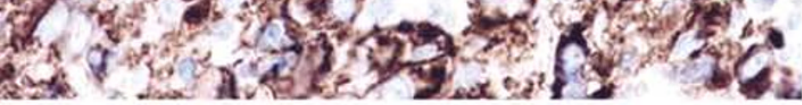

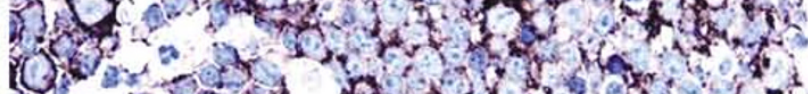

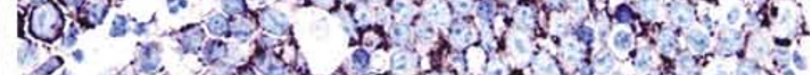

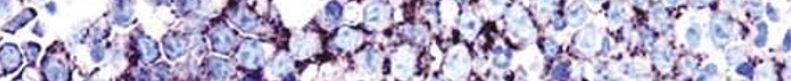
(1)

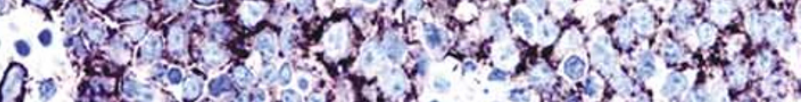

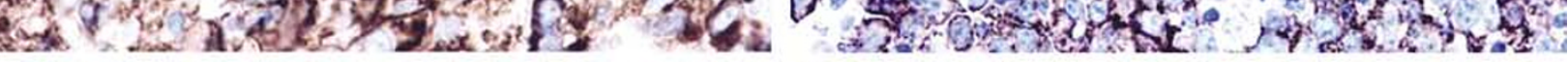

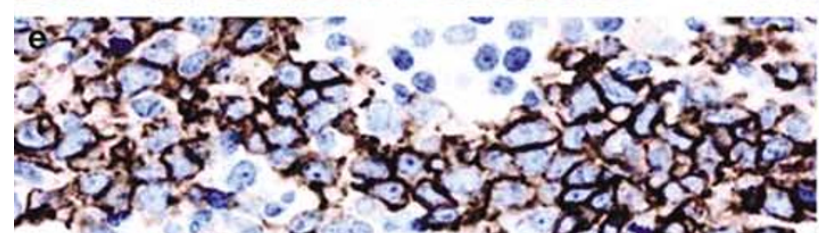

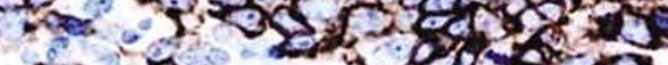
and

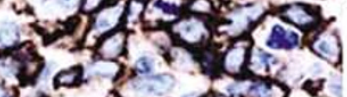

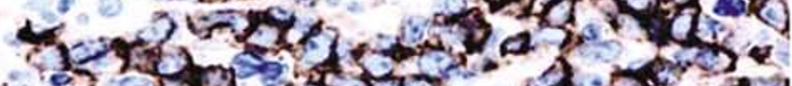
iर 30 .

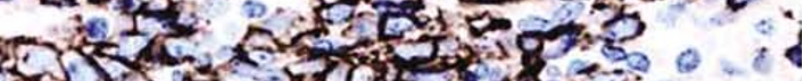

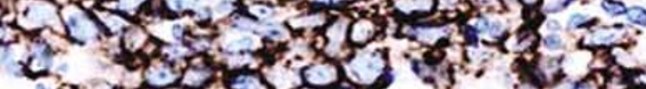

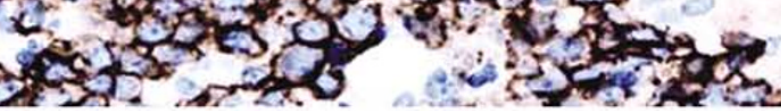
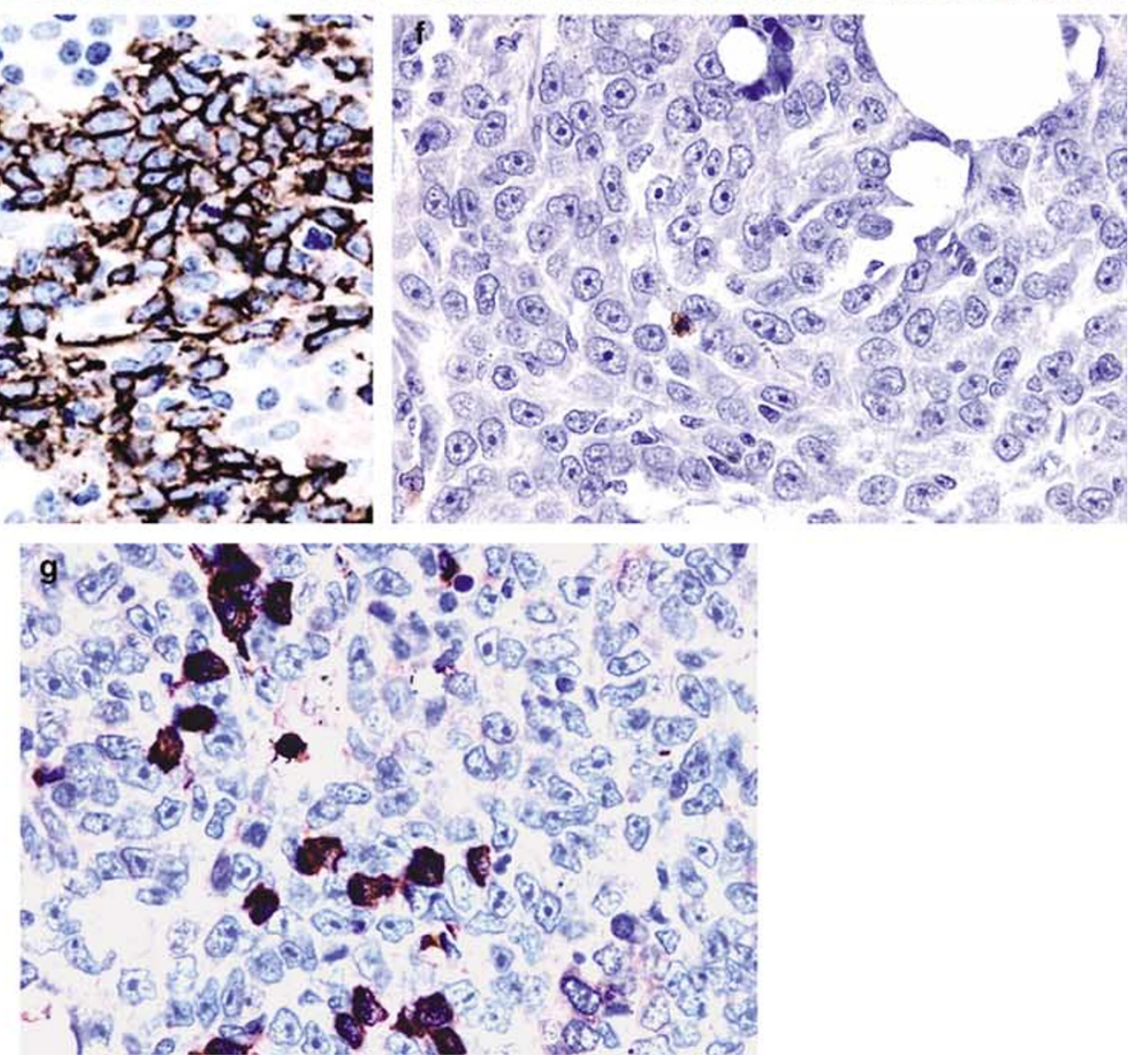
number of cases successfully analyzed with LAT was insufficient to show statistically significant difference. vWF stained only a minority of megakaryoblasts while CD31 was at times difficult to interpret for reasons that have been previously described. In AMKL, megakaryoblasts had an 'all-or-none' appearance with CD34 staining. In 7/11 cases, CD34 appeared to be diffusely positive in the vast majority of megakaryoblasts (Figure 2e), while there was almost no staining in the remaining cases (Table 2).

Three of the 11 AMKL cases were characterized by a mixture of poorly differentiated blasts, micromegakaryocytes, and larger atypical megakaryocytes. These cases did morphologically closely resemble APMF. However, immunohistochemistry showed a significantly higher frequency of CD42b- and LATpositive megakaryoblasts in these three cases than that observed in the APMF group. Reticulin fibrosis was of a similar degree both in APMF and AMKL cases.

\section{Flow Results}

Flow cytometric analysis was performed in four cases of APMF and seven cases of AMKL (Table 3).

Table 3 Flow cytometry results

Entity $\begin{gathered}\text { Case Results } \\ \text { number }\end{gathered}$

\begin{tabular}{lrl}
\hline APMF & 1 & HLA-DR, CD117, CD33, CD34, CD13, \\
& 2 & HLA-DR, CD34, CD117, CD33, CD15, CD38, CD61 \\
& 3 & $\begin{array}{l}\text { HLA-DR, CD33, CD34, CD117, CD13 CD7, CD15, } \\
\text { myeloperoxidase }\end{array}$ \\
& 4 & CD13, CD117, CD7, CD34 \\
AMKL & 7 & CD13, CD33, CD34, CD41, CD61 \\
& 9 & CD7, CD13, CD33, CD41, CD61 \\
& 10 & HLA-DR, CD11b, CD33(dim), CD4(dim), CD41, CD61 \\
& 11 & CD33, CD41, CD61 \\
& 12 & HLA-DR, CD34, CD117, CD33(dim), CD41, CD61 \\
& 15 & CD33, CD34, CD15, CD11b, CD11c, CD41 \\
& 17 & CD34, HLA-DR, CD117, CD11b, CD41, CD61
\end{tabular}

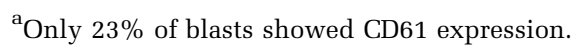

The percentage of blasts varied between 3 and $30 \%$. All four cases of APMF showed a distinct population of myeloid blasts positive for CD34, CD33, and CD117. The coexpression of early antigens and markers seen late in the myeloid maturation was seen in both APMF and AMKL. It included four cases with the coexpression of CD34 and/or CD117, and CD15, CD11b and/or CD11c. Additionally, three cases showed the aberrant coexpression of CD7 antigen. In only one case of APMF, a proportion of blasts $(23 \%)$ was positive for CD61 antigen. In contrast, all seven cases of AMKL expressed megakaryocytic markers, CD41 and/or CD61, in the majority of the blasts.

\section{Cytogenetic Results}

The results are summarized in Table 4. Three of the six patients classified as APMF had cytogenetic data. All three had evidence of partial loss of chromosome 5q. Two of the three also demonstrated monosomy of chromosome 7 or deletion of $7 \mathrm{q}$. One patient had $\operatorname{del}(5 q), \operatorname{del}(7 q)$ and trisomy 8 . All case karyotypes were consistent with a malignant myeloid disorder cytogenetically similar to aggressive MDS or MDS-related acute myeloid leukemia. Two of 11 patients classified as AMKL had cytogenetic results, and both displayed an abnormal karyotype. One had monosomy for chromosome 7 plus additional abnormalities. The second case had an interstitial deletion of the long arm of chromosome 11 between q13 and q23.

\section{Discussion}

The true nature of acute panmyelosis with myelofibrosis is not completely understood. Some believe that it is a variant of AML, and consider it to be equivalent to AMKL. ${ }^{8,18-21}$ Yet others believe that it is an acute variant of MDS, ${ }^{22-24}$ a group of disorders in which myelofibrosis can also be observed. ${ }^{25,26}$ The primary differential diagnosis of APMF is, however, with AMKL.

Table 4 Cytogenetic results

APMF Patient \#:

1. BM: 43,XY,del(5)(q13q33),-7,ins(11;?)(q13;?),-17,add(18)(q21.3), -20,?dic(20;21)(q11.2;p11.2) [6].

2. BM: Insufficient metaphases $(46, \mathrm{XX}[2])$.

4. BM: 46-48,XY,del(5)(q15q13),del(6)(q21q25), del(7)(q22q36), $\pm 8, \operatorname{del}(11)(p 11.2 p 15),-13$, add(19)(p13.3), \pm marker chromosomes [ср16].

5. BM: Insufficient metaphases (46,XY [1]).

6. BM: 46,XY,del(5)(q31q35) [3] $]^{\text {a }}$.

AMKL Patients \#:

11. BM: 46,XX,del(11)(q13q23) [16].

13. Unstimulated peripheral blood: 45,XY,-7, $\operatorname{del}(9)(q 12 q 34)[20]$ BM core: 45,XY,-7,del(9)(q12q34) [1].

${ }^{\mathrm{a}}$ In addition to $5 \mathrm{q}-$, monosomy 7 was detected by FISH on a previously obtained unstimulated peripheral blood sample.

BM: bone marrow aspirate sample. 
In both APMF and AMKL, a significant number of cases have inaspirable marrow, thereby limiting the role of cytogenetics and flow cytometry in the characterization of these diseases.

The few studies that are available do not indicate that there is a characteristic cytogenetic abnormality in cases called acute myelofibrosis. ${ }^{27-30}$ However, in cases in which a specimen sufficient for cytogenetics is obtained, complex abnormalities involving chromosome 5 and/or 7 are often seen. ${ }^{6,27-30}$

Cases of AMKL are cytogenetically more heterogeneous, and may include abnormalities of $5 / 5 q$, 7/7q, or inv(3)(q21q26). Constitutional trisomy 21 as well as $\mathrm{t}(1 ; 22)(\mathrm{p} 13 ; \mathrm{q} 13)$ can be seen in pediatric patients. ${ }^{31}$ In our series, when an adequate karyotype was obtained, overlaps were observed between the two diagnostic groups; APMF cases displayed a high incidence of complex karyotypes involving both chromosome 5 or 7 , similar to MDS and secondary AML.

Morphologically, the two disorders are known to show overlapping characteristics. Most of the past evidence, however, was based on cytologic analysis of blasts and abnormal megakaryocytic forms, as seen in smears or touch preparations, an approach that does not allow for a clear distinction between the two conditions. Besides providing a superior way to analyze megakaryocytic cells morphology and blast cell distribution, bone marrow biopsy overcomes difficulties connected to the absence or scarcity of marrow aspirate material in these myelofibrotic conditions. Research during the past decade has also shown that immunohistochemical studies of routinely processed bone marrow biopsies may be used to provide precise diagnostic information in neoplastic myeloid disorders. ${ }^{32-35}$ The identification of megakaryoblasts and atypical megakaryocytic forms can be greatly facilitated by the use of monoclonal antibodies reactive with platelet glycoproteins, such as CD41 (glycoprotein IIb/IIIa), CD42 (glycoprotein Ib), or CD61 (glycoprotein IIIa), or with a polyclonal antifactor VIII (vWF) antibody. ${ }^{36-39}$ However, of the three antiplatelet glycoprotein antibodies, only CD42 and CD61 are demonstrable in routinely processed tissue sections. Since CD61 reacts poorly in B5-fixed and/or nitric acid decalcified bone marrow biopsies, vWF has usually been the antibody of choice, at least in the United States. ${ }^{37,40}$ However, published evidence has shown that vWF expression is frequently decreased in cases of AMKL with poorly differentiated blasts, ${ }^{6}$ a finding consistent with the decreased synthesis of alpha granules (where vWF is stored) in immature megakaryocytes.

Our study of bone marrow biopsies demonstrates important differences in the morphologic and immunohistochemical characteristics of the two conditions (APMF and AMKL) that can be useful in their differential diagnosis.

The bone marrow biopsy showed a significantly higher frequency of bone marrow blasts in AMKL than in APMF. In addition, the blasts in all cases of AMKL stained strongly for CD42b (and LAT) and were negative with myeloperoxidase, a result which is consistent with their predominant megakaryocytic nature and paralleled the flow cytometric results (ie positivity with CD61 and CD41 in the majority of the blasts), available, however, in only a minority of the cases. In addition, CD34 positivity in blasts was seen in $60 \%$ of the AMKL cases in this series. In the AMKL-CD34-positive cases, the blasts coexpressed CD34 and megakaryocytic antigens.

In contrast, APMF was characterized by a less numerous population of blasts within a polymorphic cellular background, which included erythroblasts, megakaryocytes, and maturing myeloid elements with variability in their relative proportions from case to case. The blasts in APMF did not express significant megakaryocytic reactivity, but were identifiable by their strong reactivity with CD34 and occasional reactivity with myeloperoxidase. Our results thus emphasize the panmyelotic nature of APMF, as perceived by its heterogeneous morphology and immunohistochemical reactivity.

We found that CD42b is a better marker than vWF for detecting megakaryoblasts, as shown by our results. Recently, LAT (linker for activation of T-cells), a molecule involved in T-cell activation and platelet aggregation, was found to stain megakaryocytes and platelets in tissue sections ${ }^{40,41}$ as well as megakaryoblasts in cases of AMKL. ${ }^{40,41}$ In our study, LAT showed a sensitivity comparable to CD42b. However, the staining was unsatisfactory in a proportion of the cases. To confirm the practical usefulness of this marker, additional studies will be required.

In comparison to CD42b, CD31 and vWF immunostains were less effective in separating APMF from AMKL, and the differences observed did not reach a level of statistical significance. Both antibodies resulted often in a high nonspecific background staining; diffuse staining of vascular endothelium and macrophage staining with CD31 was also encountered. These markers should not be considered as 'first choice' to stain megakaryocytes in routinely processed tissue sections.

The concept that APMF represents a 'multilineage' disease characterized by a heterogeneous blast cell population has been repeatedly proposed in numerous publications over the last 40 years. In 1981, Sultan et $a l^{22}$ described several cases of acute myelofibrosis in which they were able to demonstrate a heterogeneous blast cell population, which included megakaryoblasts, poorly differentiated myeloid blasts, and erythroblasts. In 1985, Schreeder et $a l^{42}$ described two cases of acute myelofibrosis in which the majority of the blasts express myelomonocytic antigens. Only a minority of the blasts in their study expressed evidence of megakaryoblastic differentiation. More recently, Hruban et $a l^{18}$ have described in four cases of APMF studied by an immunoperoxidase technique, a complete lack of 
blast cell reactivity with vWF antibody, a result which is largely in agreement with our findings. The cases were characterized by faint staining of the blasts with lysozyme, a marker of myelomonocytic differentiation. By contrast, blast cells in three cases of AMKL studied by the same authors were positive with vWF and negative with lysozyme.

The notion that the blast cells in APMF are primitive hematopoietic cells arises from cases of this disorder that have terminated with a frankly leukemic phase characterized by myeloid or less frequently a lymphoblastic phenotype..$^{7,43,44}$ In contrast to what has been observed in cases of chronic idiopathic myelofibrosis, the 'progression' from APMF to a frank AMKL has not been well documented.

Other conditions besides AMKL need also to be considered in the differential diagnosis of APMF. These include: cases of acute leukemia (other than AMKL) with marrow fibrosis, particularly AML with multilineage dysplasia (AML-MLD), MDS with fibrosis, CIMF, and metastatic tumors with an associated myelofibrotic reaction.

The distinction between APMF and AML with myelofibrosis, particularly AML with multilineage dysplasia may occasionally be problematic. $^{45}$ Whether the distinction of these conditions is relevant for clinical management is still not clear. When the proliferative process is predominantly blastic, the case should be classified as AML $\left( \pm\right.$ MLD) with associated fibrosis. ${ }^{6}$ However, cases of AML-MLD, which occur after a prior MDS or with a therapy-related origin, ${ }^{6,33}$ frequently have a low (ie $<30 \%$ ) blast count and may closely resemble APMF or be totally morphologically indistinguishable from it.

The distinction between APMF and CIMF is usually much less problematic. In CIMF, the peculiar cytologic characteristics of the megakaryocytes, which include anisocytosis with a predominance of large size forms arranged into tight cellular clusters, the abnormal chromatin clumping with hyperchromatic nuclei and clumped (cloud shape) nuclear lobulation as well as the characteristic stromal changes that include the presence of intrasinusoidal hematopoiesis allow for a distinction with APMF. ${ }^{46}$ These characteristics usually allow for a distinction, even in the rare cases of CIMF in accelerated phase ${ }^{46}$ in which, as opposed to the 'stable' phase of the disease, an increased number of CD34-positive blasts can be observed in the bone marrow biopsy. Further findings seen in CIMF, which are not observed in APMF, include a chronic course, characteristic peripheral blood changes (ie dacrocytosis and leukoerythroblastosis), and the presence of significant splenomegaly. The latter findings may, however, be lacking in the early cellular phase of the disease.

The distinction between APMF and aggressive subtypes of MDS with fibrosis (MDS-F) ${ }^{25,33}$ such as refractory anemia with excess of blasts type 2- with fibrosis, in particular, may be difficult, as also illustrated by our case 3 . These difficulties have been outlined in published reports, ${ }^{22,47,48}$ One of the reason concerns the degree of myelofibrosis which, in both conditions, shows significant overlap. However, important clinical differences exist between the two conditions, particularly the abrupt onset for APMF and its shorter survival rate in comparison with MDS-F. ${ }^{22,25}$ The latter condition appears clinically heterogeneous and at least in one study has been associated with comparatively long survivals. $^{49}$ It seems therefore prudent to retain the diagnostic terms separately, and to consider APMF a variant of AML as defined by the WHO system. Lastly, as briefly mentioned, it is particularly important to rule out the history of exposure to leukemogenic therapeutic agents (eg post alkylating agents) or environmental toxins before diagnosing APMF. These secondary cases should be more properly termed MDS- or AML-therapy-related, and not APMF.

In summary, we have attempted to provide a comprehensive description of the pathologic findings in APMF, emphasizing its distinction from AMKL. The combination of bone marrow biopsy morphology and immunostaining permits a reproducible distinction between APMF and AMKL. Morphologic overlap can be observed between APMF, AML with myelofibrosis, and MDS-F. However, in view of important clinical implications, it seems prudent to retain the term of APMF as defined by the WHO group and above.

\section{Acknowledgements}

These results were presented in part at the 92nd Annual Meeting of the United States and Canadian Academy of Pathology, Washington, DC, 2003. We thank Professor Richard D Brunning, University of Minnesota, for his valuable advice, critical review, and suggestions on how to improve the manuscript. The authors also acknowledge Professor George Delsol, University of Toulose, France for kindly providing the anti-LAT antibody used in this study, Ms Cecilia Dobson for her invaluable technical help with immunohistochemistry, and Ms Regina D Bennett for her secretarial support.

\section{References}

1 Lewis SM, Szur L. Malignant myelosclerosis. Br Med J 1963;ii:472-477.

2 Bergsman ML, Van Slyck EJ. Acute myelofibrosis: an accelerated variant of agnogenic myeloid metaplasia. Ann Intern Med 1971;74:232-235.

3 Estevez JM, Urueta EE, Moran TJ. Acute megakaryocytic myelofibrosis. Case report of an unusual myeloproliferative syndrome. Am J Clin Pathol 1974;62:52-59. 
4 Fabich DR, Raich PC. Acute myelofibrosis: a report of three cases. Am J Clin Pathol 1977;67:334-338.

5 Bearman RM, Pangalis GA, Rappaport H. Acute (malignant) myelosclerosis. Cancer 1979;43:279-293.

6 Brunning RD, Matutes E, Flandrin G, et al. Acute myeloid leukemia not otherwise categorized-acute panmyelosis with myelofibrosis. In: Jaffe ES, Harris NL, Stein H, Vardiman JW (eds). World Health Organization Classification of Tumors, Tumors of Haematopoietic and Lymphoid Tissues. IARC Press: Lyon, France, 2001, pp 103-104.

7 Maj JS, Roslan K, Fic-Sikorska B. Acute myelofibrosis in children: report on two cases. Acta Haematol Polonica 1996;27:79-84.

8 Weisenburger DD. Acute myelofibrosis terminating as acute myeloblastic leukemia. Am J Clin Pathol 1980; 73:128-132.

9 Dunphy $\mathrm{CH}$, Kitchen S, Saravia O, et al. Acute myelofibrosis terminating in acute lymphoblastic leukemia: case report and review of the literature. Am J Hematol 1996;51:85-89.

10 Bain BJ, Catovsky D, O’Brien M, et al. Megakaryoblastic leukemia presenting as acute myelofibrosis-a study of four cases with the platelet-peroxidase reaction. Blood 1981;58:206-213.

11 Brunning RD, Matutes E, Flandrin G, et al. Acute myeloid leukemia not otherwise categorized-acute megakaryoblastic leukemia. In: Jaffe ES, Harris NL, Stein H, Vardiman JW (eds). World Health Organization Classification of Tumors, Tumors of Haematopoietic and Lymphoid Tissues. IARC Press: Lyon, France, 2001, p 99.

12 Bennett JM, Catovsky D, Daniel MT, et al. Criteria for the diagnosis of acute leukemia of megakaryocyte lineage (M7). Ann Intern Med 1985;103:460-462.

13 Breton-Gorius J, Vainchenker W. Expression of platelet proteins during the in vitro and in vivo differentiation of megakaryocytes and morphological aspects of their maturation. Semin Hematol 1986;23:43-67.

14 Fox SB, Lorenzen J, Heryet A, et al. Megakaryocytes in myelodysplasia: an immunohistochemical study on bone marrow trephines. Histopathol 1990;17: 69-74.

15 Thiele J, Quitmann H, Wagner S, et al. Dysmegakaryopoiesis in myelodysplastic syndromes (MDS): an immunomorphometric study of bone marrow trephine biopsy specimens. J Clin Pathol 1991;44:300-305.

16 Manoharan A, Horsley R, Pitney WR. The reticulin content of bone marrow in acute leukaemia in adults. Br J Haematol 1979;43:185-190.

17 Palmer CG, Blouin JL, Bull MJ, et al. Cytogenetics and molecular analysis of a ring (21) in a patient with partial trisomy 21 and megakaryocytic leukemia. Am J Med Genet 1995;57:527-536.

18 Hruban RH, Kuhajda FP, Mann RB. Acute myelofibrosis. Immunohistochemical study of four cases and comparison with acute megakaryocytic leukemia. Am J Clin Pathol 1987;88:578-588.

19 Amberger DM, Saleem A, Kemp BL, et al. Acute myelofibrosis-a leukemia of pluripotent stem cell. Ann Clin Lab Sci 1990;20:409-414.

20 Bird T, Proctor SJ. Malignant myelosclerosis: myeloproliferative disorder or leukemia. Am J Clin Pathol 1977;67:512-520.

21 den Ottolander GJ, te Velde J, Brederoo $\mathrm{P}$, et al. Megakaryoblastic leukaemia (acute myelofibrosis): a report of three cases. Br J Haematol 1979;42:9.
22 Sultan C, Sigaux F, Imbert M, et al. Acute myelodysplasia with myelofibrosis; a report of eight cases. Br J Haematol 1981;49:11-16.

23 Imbert M, Nguyen D, Sultan C. Myelodysplastic syndromes (MDS) and acute myeloid leukemias (AML) with myelofibrosis. Leukemia Res 1992;16: 51-54.

24 Allen EF, Lunde JH, McNally R, et al. A case of acute myelofibrosis with complex karyotypic changes: a type of myelodysplastic syndrome. Cancer Gene Cytogene 1996;90:24-28.

25 Lambertenghi-Deliliers G, Orazi A, Luksch R, et al. Myelodysplastic syndrome with increased marrow fibrosis: a distinct clinicopathological entity. $\mathrm{Br} \mathrm{J}$ Haematol 1991;78:161-166.

26 Maschek H, Georgii A, Kaloutsi V, et al. Myelofibrosis in primary myelodysplastic syndromes: a retrospective study of 352 patients. Eur J Haematol 1992; 48:208-214.

27 Mitus WF, Coleman N, Kiossoglou KA. Abnormal (Marker) chromosomes in two patients with acute myelofibrosis. Arch Intern Med 1969;123:192.

28 Van Slyck EJ, Weiss L, Dully M. Chromosomal evidence for the secondary role of fibroblastic proliferation in acute myelofibrosis. Blood 1970;36: 729-735.

29 Nowell P, Jensen J, Gardner F, et al. Chromosome studies in 'preleukemia' III. Myelofibrosis. Cancer 1976;38:1873-1881.

30 Shah I, Mayeda K, Koppitch F, et al. Karyotypic polymorphism in acute myelofibrosis. Blood 1982;60: 841-844.

31 Cuneo A, Mecucci C, Kerim S, et al. Multipotent stem cell involvement in megakaryoblastic leukemia: cytologic and cytogenetic evidence in 15 patients. Blood 1989;74:1781-1790.

32 Soligo D, Delia D, Oriani A, et al. Identification of CD34+cells in normal and pathological bone marrow biopsies by QBEND10 monoclonal antibody. Leukemia 1991;12:1026-1030.

33 Orazi A, Cattoretti G, Soligo D, et al. Therapyrelated myelodysplastic syndromes: FAB classification, bone marrow histology, and immunohistology in the prognostic assessment. Leukemia 1993;7: 838-847.

34 Orazi A, Cattoretti G, Heerema NA, et al. Frequent p53 overexpression in therapy related myelodysplastic syndromes and acute myeloid leukemias: an immunohistochemical study of bone marrow biopsies. Mod Pathol 1993;6:521-525.

35 Orazi A, Neiman RS, Cualing H, et al. CD34 immunostaining of bone marrow biopsy specimens is a reliable way to classify the phases of chronic myeloid leukemia. Am J Clin Pathol 1994;101:426-468.

36 Erber WN, Breton-Gorius J, Villeval JL, et al. Detection of cells of megakaryocyte lineage in haematological malignancies by immuno-alkaline phosphatase labelling cell smears with a panel of monoclonal antibodies. Br J Haematol 1987;65:87-94.

37 Chuang SS, Li CY. Useful panel of antibodies for the classification of acute leukemia by immunohistochemical methods in bone marrow trephine biopsy specimens. Am J Clin Pathol 1997;107:410-418.

38 Thiele J, Krech R, Wienhold S, et al. The use of the anti-factor VIII method on trephine biopsies of the bone marrow for the identification of immature and atypical megakaryocytes in myeloproliferative 
diseases and allied disorders. A morphometric study. Virchows Arch B Cell Pathol 1987;54:89-97.

39 Thiele J, Quitmann H, Wagner S, et al. Dysmegakaryopoiesis in myelodysplastic syndromes (MDS): an immunomorphometric study of bone marrow trephine biopsy specimens. J Clin Pathol 1991;44:300-305.

40 Facchetti F, Chan JK, Zhang W, et al. Linker for activation of $\mathrm{T}$ cells (LAT), a novel immunohistochemical marker for $\mathrm{T}$ cells, $\mathrm{NK}$ cells, mast cells, and megakaryocytes: evaluation in normal and pathological conditions. Am J Pathol 1999;154: 1037-1046.

41 Ungari M, Pellegrini W, Borlenghi E, et al. LAT (linker for activation of $\mathrm{T}$ cells): a useful marker for megakaryocyte evaluation on bone marrow biopsies. Patologica 2002;94:325-330.

42 Schreeder MT, Prchal JT, Parmley RT, et al. An acute myeloproliferative disorder characterized by myelofibrosis and blast cells that express phenotypic properties associated with multiple hematopoietic lineages. Am J Clin Pathol 1985;83:114-121.

43 Amjad H, Gezer S, Inoue S, et al. Acute myelofibrosis terminating in an acute lymphoblastic leukemia. Cancer 1980;46:615-618.
44 Puckett JB, Cooper MR. Acute myelofibrosis evolving into acute myeloblastic leukemia. Ann Inter Med 1981;94:545-546.

45 Brunning RD, Matutes E, Harris NL, et al. Acute myeloid leukemia with multilineage dysplasia. In: Jaffe ES, Harris NL, Stein H, Vardiman, JW (eds). World Health Organization Classification of Tumors, Tumors of Haematopoietic and Lymphoid Tissues. IARC Press: Lyon, France, 2001, pp 88-89.

46 Thiele J, Pierre $\mathrm{R}$, Imbert $\mathrm{M}$, et al. Chronic idiopathic myelofibrosis. In: Jaffe ES, Harris NL, Stein H, Vardiman JW (eds). World Health Organization Classification of Tumors, Tumors of Haematopoietic and Lymphoid Tissues. IARC Press: Lyon, France, 2001, p 35.

47 Thiele J, Kvasnika HM, Schmitt-Graeff A. Acute panmyelosis with myelofibrosis. Leuk Lymphoma 2004;45:681-687.

48 Steensma DP, Hanson CA, Letendre L, et al. Myelodysplasia with fibrosis: a distinct entity? Leuk Res 2001;25:829-838.

49 Pagliuca A, Layton DM, Manoharan A, et al. Myelofibrosis in primary myelodysplastic syndromes: a clinico-morphological study of 10 cases. Br J Haematol 1989;71:499-504. 